\title{
Pretreatment malnutrition and quality of life - association with prolonged length of hospital stay among patients with gynecological cancer: a cohort study
}

\author{
Brenda Laky1,4, Monika Janda2, Srinivas Kondalsamy-Chennakesavan+4, Geoffrey Cleghorn 3,4 and Andreas Obermair*1,4
}

\begin{abstract}
Background: Length of hospital stay (LOS) is a surrogate marker for patients' well-being during hospital treatment and is associated with health care costs. Identifying pretreatment factors associated with LOS in surgical patients may enable early intervention in order to reduce postoperative LOS.

Methods: This cohort study enrolled 157 patients with suspected or proven gynecological cancer at a tertiary cancer centre (2004-2006). Before commencing treatment, the scored Patient Generated - Subjective Global Assessment (PGSGA) measuring nutritional status and the Functional Assessment of Cancer Therapy-General (FACT-G) scale measuring quality of life (QOL) were completed. Clinical and demographic patient characteristics were prospectively obtained. Patients were grouped into those with prolonged LOS if their hospital stay was greater than the median LOS and those with average or below average LOS.

Results: Patients' mean age was 58 years (SD 14 years). Preoperatively, 81 (52\%) patients presented with suspected benign disease/pelvic mass, 23 (15\%) with suspected advanced ovarian cancer, 36 (23\%) patients with suspected endometrial and $17(11 \%)$ with cervical cancer, respectively. In univariate models prolonged LOS was associated with low serum albumin or hemoglobin, malnutrition (PG-SGA score and PG-SGA group B or C), low pretreatment FACT-G score, and suspected diagnosis of cancer. In multivariable models, PG-SGA group B or C, FACT-G score and suspected diagnosis of advanced ovarian cancer independently predicted LOS.

Conclusions: Malnutrition, low quality of life scores and being diagnosed with advanced ovarian cancer are the major determinants of prolonged LOS amongst gynecological cancer patients. Interventions addressing malnutrition and poor QOL may decrease LOS in gynecological cancer patients.
\end{abstract}

\section{Background}

Despite significant advances in cancer therapy, in-patient hospital care remains a major expense in the treatment of patients with gynecological cancer. Reducing length of hospital stay (LOS) has the potential to decrease health care cost, risk of infections and other hospital acquired diseases, and to improve patients' quality of life (QOL). Previous studies have observed differential LOS by cancer type and stage, however, these characteristics commonly are not available before surgery, and thus are not

*Correspondence: andreas_obermair@health.qld.gov.au

${ }^{1}$ Queensland Centre for Gynaecological Cancer, Level 6 Ned Hanlon Building, The Royal Brisbane and Women's Hospital, Herston Queensland 4029, Australia Full list of author information is available at the end of the article amenable to pre-treatment interventions. Identifying modifiable risk factors at admission predicting (LOS) could lead to appropriately targeted interventions to improve the delivery of care for women with gynecological cancer. Previous research has shown that preoperative serum albumin (a marker of chronic malnutrition), hemoglobin and lymphocyte status are associated with LOS and mortality in the gynecological oncology setting [1-4]. Preoperative medical conditions or co-morbidities could also be associated with LOS; however findings regarding these predictors are inconsistent $[4,5]$.

Acute malnutrition may be a further potentially amenable risk factor for LOS. Malnutrition has been found to be 
associated with increased risk of morbidity and mortality, complication rates such as wound infections, costs of hospitalisation, and decreased QOL in various cancer populations [2,4,6-16]. The scored Patient Generated Subjective Global Assessment (PG-SGA) is a validated nutritional assessment tool specifically designed for cancer patients. Using the PG-SGA, we have observed high levels of nutritional deficiencies among patients with gynecological cancer, especially those diagnosed with ovarian cancer $[17,18]$, but to date no study has assessed whether the scored PG-SGA can predict prolonged LOS among gynecological cancer patients.

Gynecological cancer and/or malnutrition can have a profound impact on patients' physical function and psychosocial well-being - both important components of QOL. One common definition of QOL is that it is a subjective, multidimensional construct representing functional status, mental and social well-being as well as general health [19]. Health-related QOL before treatment commences has been suggested as an important prognostic factor in ovarian cancer patients [20,21]. To our knowledge, no study to date evaluated the association between preoperative QOL, malnutrition and LOS among a wider range of gynecological cancer patients.

Thus, the purpose of this study was to evaluate factors available prior to initial treatment to predict LOS in patients with suspected or proven gynecological cancer.

\section{Methods}

The study was conducted at the Queensland Centre for Gynecological Cancer at the Royal Brisbane and Women's Hospital, Brisbane, Australia. Patients with presumed or proven primary gynecological cancer were screened for eligibility at their preoperative visit between March 2004 and December 2006. Patients were excluded from the study if they presented with recurrent cancer, had received treatment for other cancers within the past five years, had psychological or cognitive impairments (e.g. schizophrenia, dementia) or were non-English speaking. A total of 194 patients gave informed written consent. Thirty-two study participants declined to complete the Functional Assessment of Cancer Therapy-General (FACT-G) questionnaire and five participants had to be excluded from analyses due to incomplete data. Overall, 157 women completed both the scored PG-SGA and the FACT-G questionnaire before commencing treatment. Patients' clinical and demographic characteristics including age at study entry, body mass index, serum albumin, hemoglobin, lymphocytes, co-morbidities, adverse events, surgical approach, LOS, and histopathological diagnosis and staging according to the International Federation of Gynecology and Obstetrics (FIGO) were recorded prospectively. Six women did not undergo surgery, because a surgical approach was contraindicated or chemotherapy and/or radiotherapy was initiated as the primary treatment. Pre-treatment serum albumin levels were recorded in 146 patients; hemoglobin and lymphocyte count in 153 patients and actual body weight and height for all but one patient. All pre-treatment predictive factors were collected either at the outpatient or preadmission clinic, typically one to five weeks before primary treatment was initiated. The median waiting time for surgery was two weeks for women with suspected ovarian and cervical cancers and five weeks for women with suspected benign diseases or endometrial cancer, respectively. Women were categorised according to their clinical diagnosis before surgery: pelvic mass/ benign disease, suspected advanced ovarian cancer, cervical cancer or endometrial cancer. This study has been approved by The Royal Brisbane and Women's Hospital Human Research Ethics Committee (Protocol Number 2004/007) and the University of Queensland Medical Research Ethics Committee (Brisbane, Australia; Project Number 2006000533).

\section{Nutritional assessment}

The scored PG-SGA is a validated nutritional assessment tool for cancer patients $[9,16]$ that records and summarizes weight changes, alterations in food intake, gastrointestinal symptoms (such as nausea, vomiting and diarrhoea that have persisted for two weeks), and changes in functional capacity and physical signs of malnutrition. These signs were assessed by a trained clinical dietician using skinfold measurements (loss of subcutaneous fat, muscle wasting, edema). The presence of ascites was established radiologically and abstracted from the clinical notes for this study. Based on the global rating, women were classified as well nourished (PG-SGA A); moderately malnourished or suspected of being malnourished (PG-SGA B); or severely malnourished (PG-SGA C). The PG-SGA also incorporates a numerical score (PG-SGA score). Typical scores achieved by gynaecological cancer patients range from 0-28 [22], with higher scores reflecting greater risk of malnutrition. Scores of nine or more indicate a critical need for nutritional intervention options and/or improved symptom management [23].

\section{QOL assessment}

QOL was measured by the FACT-G questionnaire, which is a widely utilized and validated instrument [24]. Version 4 of the FACT-G is a 27 -item self-report measure and allows patients to rate their current physical ( 7 items, range: 0-28), social and family (7 items, range: $0-28$ ), emotional (6 items, range: 0-24), and functional wellbeing (7 items, range: 0-28). The FACT-G is scored using a 5 -point scale $(0=$ not at all, $1=$ a little bit, $2=$ somewhat, 3 = quite a bit, $4=$ very much). The raw scores were 
are linearly transformed as per questionnaire manual with higher scores indicating better QOL [25].

\section{Statistical analysis}

Descriptive statistics were used to summarize patient characteristics and to group patients by the main outcome variable (average versus prolonged LOS). Prolonged LOS was defined as LOS of greater than five days for patients who underwent open abdominal surgery and greater than two days for patients who underwent vaginal of laparoscopic surgery. Univariate logistic regression analyses were conducted to calculate odds ratios adjusted for age and surgical approach for patients who had prolonged compared to average LOS.

Variables significantly associated with LOS were then entered into a multivariable model (Table 1).

All models were adjusted for age and surgical approach (open-abdominal, vaginal or laparoscopic surgery). Hosmer-Lemeshow goodness-of-fit statistic assessed fit of the models. SPSS software version 16.0 Graduate Student (SPSS Inc., Chicago, IL, USA) was used for the statistical analyses.

\section{Results}

\section{Patient Characteristics}

Patients' mean age was 58 years (SD 14 years). Preoperatively, 81 (52\%) patients presented with suspected benign disease/pelvic mass, 23 (15\%) were suspected to have advanced ovarian cancer, 36 (23\%) patients to have endometrial cancer, and 17 (11\%) patients to have cervical cancer. Ninety-four patients were operated using an open-abdominal, 40 patients with a laparoscopic and 17 patients with a vaginal surgical approach. Data on patients' weight, body mass index, albumin and PG-SGA have been reported in previous publications $[17,22]$.

\section{LOS and potential predictors}

The median LOS for all patients in the study was 5 days (range 0-43 days). In total, 75 (48.1\%) of the patients had prolonged LOS (Table 1). Patients with prolonged LOS were significantly older (mean age 62.8 years, SD 13.7) than patients with average LOS (mean age 54.5 years, SD 13.6) $(\mathrm{p}<0.01)$.

BMI was not significantly associated with LOS. Although fifty patients had two or more pre-existing medical co-morbidities, neither the number (Table 1), nor the specific type of co-morbidity (data not shown), were significantly different between patients with average and prolonged LOS. Low pretreatment serum albumin or hemoglobin levels, low QOL FACT-G scores and high PG-SGA scores were associated with prolonged LOS. Compared to patients with a suspicious pelvic mass, patients suspected to carry an advanced ovarian cancer were significantly more likely to have prolonged LOS (OR $=30.4 ; 95 \% \mathrm{CI}=3.9-236.7)($ Table 1$)$.

In the multivariable model, patients with good QOL were at significantly lower risk for prolonger LOS (OR = 0.96, 95\% CI 0.93-0.99; $\mathrm{p}=0.007)$. Patients with PG-SGA $\mathrm{B}$ or $\mathrm{C}(\mathrm{OR}=5.28,95 \% \mathrm{CI}=0.98-28.5, \mathrm{p}=0.05)$ and patients with suspected advanced stage ovarian cancer $(\mathrm{OR}=14.7,95 \% \mathrm{CI}=1.61-134.7 . \mathrm{p}=0.02)$ were significantly more likely to have prolonger LOS compared to other patients (Table 1).

\section{Discussion}

This study assessed factors predictive for prolonged LOS (defined as $>5$ days or $>2$ days, for patients who had open abdominal or laparoscopic surgery, respectively) among gynecological cancer patients. Malnutrition and low QOL were found to predict prolonged LOS independent of patients' age at diagnosis, surgical approach (laparoscopy or laparotomy), albumin and hemoglobin and suspected clinical diagnosis. Patients suspected to have stage III or IV ovarian cancer were also independently at greater risk for prolonged LOS compared to other patients.

Previous studies have shown that malnutrition was associated with prolonged LOS in hospitalized patients requiring treatment for various types of cancer $[13,16,26$ 28]. The present manuscript is the first to confirm those findings for patients with gynecological cancer, and extends these findings even further. The present data suggest an association between pre-operative malnutrition and LOS based on both the PG-SGA global rating and the PG-SGA score. To our knowledge there is only one previous study which assessed the PG-SGA score (as opposed to the global rating) and LOS [15]. Thomas and colleagues found a weak correlation between LOS and the PG-SGA score [15], and similar in our study the PG-SGA score was the weaker predictor compared to the global rating in multivariable analysis. However, the PG-SGA score provides dieticians with suggested appropriate intervention strategies and thus can be a clinically useful adjunct to the global rating.

Our findings show that LOS was not equally distributed between tumor types. Even after adjustment for age and surgical approach, $96 \%$ of all suspected advanced stage ovarian cancer patients had prolonged hospital stay, compared with only $50 \%$ of patients with endometrial cancer and $42 \%$ of patients with a pelvic mass. In our previous work we described in detail that malnutrition was almost exclusively present in ovarian cancer patients compared to other gynecologic oncology diagnoses [17].

A previous study by Dean and colleagues found that gynecological cancer patients with two or more pre-existing co-morbidities had significantly longer LOS than 
Table 1: Pre-surgical factors associated with prolonged length of hospital stay (LOS).

\begin{tabular}{|c|c|c|c|c|c|c|c|c|}
\hline & \multirow[b]{2}{*}{$\mathbf{N}$} & \multirow{2}{*}{$\begin{array}{l}\text { \% (n) with } \\
\text { prolonged LOS }\end{array}$} & \multicolumn{2}{|c|}{ Univariate model1 } & \multirow[b]{2}{*}{$P$} & \multicolumn{3}{|c|}{ Multivariable model2 } \\
\hline & & & OR & $95 \% \mathrm{Cl}$ & & OR & $95 \% \mathrm{Cl}$ & $P$ \\
\hline Total & 157 & & 48.1 & & & & & \\
\hline \multicolumn{9}{|l|}{ Body mass index } \\
\hline Underweight $(<18.5)$ & 3 & $66.7(2)$ & 2.96 & $0.12-70.3$ & 0.50 & & & \\
\hline Normal (18.5-24.99) & 39 & $46.2(18)$ & \multicolumn{3}{|c|}{ Reference group } & & & \\
\hline Overweight (25.0-29.99) & 47 & $44.7(21)$ & 0.91 & $0.36-2.30$ & 0.84 & & & \\
\hline Obese $(\geq 30)$ & 67 & $50.7(34)$ & 1.31 & $0.55-3.16$ & 0.54 & & & \\
\hline \multicolumn{9}{|l|}{ Pre-existing co-morbidity } \\
\hline No or 1 co-morbidity & 107 & $45.8(49)$ & \multicolumn{3}{|c|}{ Reference group } & & & \\
\hline 2 or more co-morbidities & 50 & $54.0(27)$ & 0.12 & $0.56-2.75$ & 0.59 & & & \\
\hline \multicolumn{9}{|l|}{ Blood } \\
\hline \multicolumn{9}{|l|}{ Albumin } \\
\hline Average (>35 g/L) & 124 & $41.9(52)$ & \multicolumn{3}{|c|}{ Reference group } & & & \\
\hline Below Average ( $\leq 35 \mathrm{~g} / \mathrm{L})$ & 22 & $86.4(19)$ & 8.81 & $1.82-42.6$ & 0.007 & 0.86 & $0.12-6.5$ & 0.88 \\
\hline \multicolumn{9}{|l|}{ Hemoglobin } \\
\hline Average (>120 g/L) & 117 & $42.7(50)$ & \multicolumn{2}{|c|}{ Reference group } & & & & \\
\hline Below Average $(\leq 120 \mathrm{~g} / \mathrm{L})$ & 36 & $69.4(25)$ & 3.21 & $1.31-7.68$ & 0.01 & 2.11 & $0.65-6.86$ & 0.21 \\
\hline \multicolumn{9}{|l|}{ Lymphocytes } \\
\hline Average (1.5-4.0 × 109/L) & 109 & $44.0(48)$ & \multicolumn{3}{|c|}{ Reference group } & & & \\
\hline Below Average $\left(\leq 1.5 \times 10^{9} / \mathrm{L}\right)$ & 44 & $64.4(27)$ & 1.46 & $0.66-3.22$ & 0.35 & & & \\
\hline FACT-G global score & 157 & {$[75.3 \pm 16.9]^{4}$} & 0.95 & $0.93-0.98$ & $<0.001$ & 0.96 & $0.93-0.99$ & 0.007 \\
\hline \multicolumn{9}{|l|}{ Nutritional assessment } \\
\hline PG-SGA score & 157 & {$[9.5 \pm 6.6]^{4}$} & 1.13 & $1.05-1.22$ & 0.001 & 0.94 & $0.84-1.06$ & 0.33 \\
\hline PG-SGA A & 118 & $39.3(44)$ & \multicolumn{2}{|c|}{ Reference group } & & & & \\
\hline PG-SGA B and C & 39 & $82.1(32)$ & 6.89 & $2.48-19.2$ & $<0.001$ & 5.28 & $0.98-28.5$ & 0.05 \\
\hline \multicolumn{9}{|l|}{ Histology and Stage } \\
\hline Benign/LMP/OvCa stage I or II & 81 & $42.0(34)$ & \multicolumn{2}{|c|}{ Reference group } & & & & \\
\hline OvCa stage III or IV & 23 & $95.7(22)$ & 30.4 & $3.9-236.7$ & 0.001 & 14.7 & $1.6-134.7$ & 0.02 \\
\hline Endometrial cancer & 36 & $50.0(18)$ & 1.38 & $0.63-3.04$ & 0.42 & 1.71 & $0.67-4.34$ & 0.07 \\
\hline Cervical cancer & 22 & $11.8(2)$ & 0.18 & $0.04-0.86$ & 0.03 & 0.10 & $0.01-0.71$ & 0.84 \\
\hline
\end{tabular}

1adjusted for age and surgical approach; ${ }^{2}$ Multivariable model including albumin, FACT-G score, PG-SGA score and PG-SGA global rating; adjusted for age and surgical approach; ${ }^{3}$ Continuous variables: mean \pm standard deviation for group with average LOS; ${ }^{4}$ Continuous variables: mean \pm standard deviation for group with prolonged LOS;

Abbreviations: LOS, Length of hospital stay; OR, Odds Ratio; Cl, Confidence interval; PG-SGA, Patient-Generated Subjective Global Assessment; FACT-G, Functional Assessment of Cancer Therapy-General.

those with one or no co-morbidities [5]. In contrast, our findings suggest that in this sample of gynecological cancer patients, neither the number of co-morbidities nor the type of co-morbidity are predictive of prolonged LOS. Further studies are required to validate these findings, carefully separating preoperative co-morbidities from postoperative complications.

In this study, we found low preoperative hemoglobin and lymphocyte counts in a small number of patients and these patients were more likely to experience prolonged LOS, but this difference was no longer statistically significant in adjusted modelling. Previous studies suggested that low hemoglobin levels were related to poor survival in patients with ovarian cancer $[29,30]$, and studies differed in their conclusions regarding the importance of age, histology, nutritional status and immune system on lymphocyte counts [31-33]. With our non-significant 
results, we suggest that neither of these markers are suitable factors to predict prolonged LOS.

A third biochemical marker assessed in the present study was serum albumin, generally believed to indicate the presence of chronic malnutrition. Previous studies have demonstrated an association between low serum albumin and increased postoperative complications $[6,34,35]$, as well as reductions in survival in ovarian cancer patients [36]. The results of our study confirmed previous findings by Massad and colleagues in which they found an inverse correlation between serum albumin and LOS among patients with gynecological cancer [4]. However, our current results demonstrated that when albumin and PG-SGA global rating [22] were assessed concurrently, the PG-SGA was a stronger predictor of LOS, and therefore more sensitive to detect malnutrition in gynecological cancer patients.

Research conducted on QOL and nutritional status in cancer patients indicates that nutritional support may lead to better QOL $[11,37,38]$. To our knowledge few investigations have examined the association between patients' pre-treatment QOL and prolonged LOS, while concurrently assessing malnutrition. One study by Rogers and colleagues found a weak correlation between better QOL and shorter LOS in oral and oropharyngeal cancer patients [39]. In the present study we found that poor QOL prior to initial treatment was associated with prolonged LOS, and this association remained significant when adjusted for other variables. Our results suggest that subsequent studies should assess the effect of presurgical nutritional interventions, or interventions aimed at improving QOL on LOS. A limitation of our study was that data on nutritional status and QOL were only assessed before commencement of initial treatment and not at any time point afterwards. This additional information would have been of interest to identify whether changes in patients' PG-SGA score and global rating and FACT-G score are more influential for LOS than pretreatment score alone. In addition, given the preponderance of malnutrition among ovarian cancer patients, subsequent studies should focus on this subgroup of gynecological cancer patients. However, our study also has some major strengths, including the availability of pre-treatment assessments for all patients, and the high participation rate among patients with gynecological cancer.

\section{Conclusions}

In conclusion, prolonged LOS was found to be associated with low presurgical QOL and malnutrition. Strategies to reduce LOS and improve patients' well-being during hospital stay will need to address these potentially modifiable factors, particularly among women with suspected advanced ovarian cancer.
Competing interests

The authors declare that they have no competing interests.

\section{Authors' contributions}

The authors' responsibilities were as follows - $\mathrm{AO}$ : initiated the study; $\mathrm{BL}, \mathrm{SK}$ : collected data; $\mathrm{AO}, \mathrm{BL}$, and $\mathrm{MJ}$ : data analysis; $\mathrm{AO}$ and $\mathrm{GC}$ : project supervision; $A O, B L, S K$ and MJ: writing the manuscript; and all authors: revision of the manuscript.

\section{Acknowledgements}

The authors gratefully acknowledge the contribution of all patients who participated in this study.

This research was funded by a National Health and Medical Research Council Career Development Award (to MJ), the Endeavour International Postgraduate Research Scholarship (Endeavour IPRS), the University of Queensland International Living Allowance Scholarship (UQILAS) (both to BL for this PhD project) and the Royal Brisbane and Women's Hospital Research Foundation (to AO).

\section{Author Details}

1 Queensland Centre for Gynaecological Cancer, Level 6 Ned Hanlon Building, The Royal Brisbane and Women's Hospital, Herston Queensland 4029, Australia , 2School of Public Health, Queensland University of Technology, Brisbane, Australia, ${ }^{3}$ Children's Nutrition Research Centre, Royal Children's Hospital, Brisbane, Australia and 4 School of Medicine, University of Queensland, Brisbane, Australia

Received: 13 October 2009 Accepted: 25 May 2010

Published: 25 May 2010

\section{References}

1. Detsky AS, Baker JP, O'Rourke K, Goel V: Perioperative parenteral nutrition: a meta-analysis. Ann Intern Med 1987, 107:195-203.

2. Santoso JT, Canada T, Latson B, Aaaadi K, Lucci JA, Coleman RL: Prognostic nutritional index in relation to hospital stay in women with gynecologic cancer. Obstet Gynecol 2000, 95:844-846.

3. Bishara S, Griffin M, Cargill A, Bali A, Gore ME, Kaye SB, Shepherd JH, Van Trappen PO: Pre-treatment white blood cell subtypes as prognostic indicators in ovarian cancer. Eur J Obstet Gynecol Reprod Biol 2007.

4. Massad LS, Vogler G, Herzog TJ, Mutch DG: Correlates of length of stay in gynecologic oncology patients undergoing inpatient surgery. Gynecol Oncol 1993, 51:214-218

5. Dean MM, Finan MA, Kline RC: Predictors of complications and hospital stay in gynecologic cancer surgery. Obstet Gynecol 2001, 97:721-724.

6. Obermair A, Hagenauer S, Tamandl D, Clayton RD, Nicklin JL, Perrin LC, Ward BG, Crandon AJ: Safety and efficacy of low anterior en bloc resection as part of cytoreductive surgery for patients with ovarian cancer. Gynecol Oncol 2001, 83:115-120.

7. Terada KY, Christen C, Roberts JA: Parenteral nutrition in gynecology. J Reprod Med 1988, 33:957-960.

8. Burnett AF, Potkul RK, Barter JF, Barnes WA, Delgado G: Colonic surgery in gynecologic oncology. Risk factor analysis. J Reprod Med 1993, 38:137-141.

9. Ottery FD: Definition of standardized nutritional assessment and interventional pathways in oncology. Nutrition 1996, 12:S15-19.

10. Montazeri A, McEwen J, Gillis CR: Quality of life in patients with ovarian cancer: current state of research. Support Care Cancer 1996, 4:169-179.

11. Ravasco P, Monteiro-Grillo I, Vidal PM, Camilo ME: Cancer: disease and nutrition are key determinants of patients' quality of life. Support Care Cancer 2004, 12:246-252

12. Gupta D, Lis CG, Granick J, Grutsch JF, Vashi PG, Lammersfeld CA: Malnutrition was associated with poor quality of life in colorectal cancer: a retrospective analysis. J Clin Epidemiol 2006, 59:704-709.

13. Horsley P, Bauer J, Gallagher B: Poor nutritional status prior to peripheral blood stem cell transplantation is associated with increased length of hospital stay. Bone Marrow Transplant 2005, 35:1113-1116.

14. Martineau J, Bauer JD, Isenring E, Cohen S: Malnutrition determined by the patient-generated subjective global assessment is associated with poor outcomes in acute stroke patients. Clin Nutr 2005, 24:1073-1077.

15. Thomas JM, Isenring E, Kellett E: Nutritional status and length of stay in patients admitted to an Acute Assessment Unit. J Hum Nutr Diet 2007, 20:320-328 
16. Bauer J, Capra S, Ferguson M: Use of the scored Patient-Generated Subjective Global Assessment (PG-SGA) as a nutrition assessment tool in patients with cancer. Eur J Clin Nutr 2002, 56:779-785.

17. Laky B, Janda M, Bauer J, Vavra C, Cleghorn G, Obermair A: Malnutrition among gynaecological cancer patients. Eur J Clin Nutr 2007, 61:642-646.

18. Laky B, Janda M, Cleghorn G, Obermair A: Comparison of different nutritional assessments and body-composition measurements in detecting malnutrition among gynecologic cancer patients. Am J Clin Nutr 2008, 87:1678-1685.

19. Cella DF: Quality of life: concepts and definition. J Pain Symptom Manage 1994, 9:186-192.

20. Gil KM, Gibbons HE, Jenison EL, Hopkins MP, von Gruenigen VE: Baseline characteristics influencing quality of life in women undergoing gynecologic oncology surgery. Health Qual Life Outcomes 2007, 5:25.

21. von Gruenigen VE, Frasure HE, Jenison EL, Hopkins MP, Gil KM: Longitudinal assessment of quality of life and lifestyle in newly diagnosed ovarian cancer patients: the roles of surgery and chemotherapy. Gynecol Oncol 2006, 103:120-126.

22. Laky B, Janda M, Cleghorn G, Obermair A: Comparison of different nutritional assessments and body composition measurements to detect malnutrition among gynaecological cancer patients. Am J Clin Nutr 2008 in press. accepted 14th March, 2008

23. Ottery FD: Patient-Generated Subjective Global Assessment. In The Clinical Guide to Oncology Nutrition Edited by: McCallum PD, Polisena CG. Chicago: The American Dietetic Association; 2000:11-23.

24. Cella DF, Tulsky DS, Gray G, Sarafian B, Linn E, Bonomi A, Silberman M, Yellen SB, Winicour P, Brannon J: The Functional Assessment of Cancer Therapy scale: development and validation of the general measure. $J$ Clin Oncol 1993, 11:570-579.

25. Cella D, Hahn EA, Dineen K: Meaningful change in cancer-specific quality of life scores: differences between improvement and worsening. Qual Life Res 2002, 11:207-221.

26. Baker JP, Detsky AS, Wesson DE, Wolman SL, Stewart S, Whitewell J, Langer B, Jeejeebhoy KN: Nutritional assessment: a comparison of clinical judgement and objective measurements. N Engl J Med 1982, 306:969-972.

27. Planas M, Audivert S, Perez-Portabella C, Burgos R, Puiggros C, Casanelles JM, Rossello J: Nutritional status among adult patients admitted to an university-affiliated hospital in Spain at the time of genoma. Clin Nutr 2004, 23:1016-1024.

28. Pichard C, Kyle UG, Morabia A, Perrier A, Vermeulen B, Unger P: Nutritional assessment: lean body mass depletion at hospital admission is associated with an increased length of stay. Am J Clin Nutr 2004 79:613-618.

29. Obermair A, Handisurya A, Kaider A, Sevelda P, Kolbl H, Gitsch G: The relationship of pretreatment serum hemoglobin level to the survival of epithelial ovarian carcinoma patients: a prospective review. Cancer 1998, 83:726-731

30. Obermair A, Petru E, Windbichler G, Peters-Engl C, Graf AH, Stummvoll W, Kaider A, Kurschel S, Kolbl H, Sevelda P: Significance of pretreatment serum hemoglobin and survival in epithelial ovarian cancer. Oncol Rep 2000, 7:639-644

31. Izaks GJ, Remarque EJ, Becker SV, Westendorp RG: Lymphocyte count and mortality risk in older persons. The Leiden 85-Plus Study. J Am Geriatr Soc 2003, 51:1461-1465.

32. Kuzuya M, Kanda S, Koike T, Suzuki Y, Iguchi A: Lack of correlation between total lymphocyte count and nutritional status in the elderly. Clin Nutr 2005, 24:427-432.

33. van Bokhorst-de Schueren MA van der, van Leeuwen PA, Sauerwein HP, Kuik DJ, Snow GB, Quak JJ: Assessment of malnutrition parameters in head and neck cancer and their relation to postoperative complications. Head Neck 1997, 19:419-425.

34. Gibbs J, Cull W, Henderson W, Daley J, Hur K, Khuri SF: Preoperative serum albumin level as a predictor of operative mortality and morbidity: results from the National VA Surgical Risk Study. Arch Surg 1999, 134:36-42

35. Alphs HH, Zahurak ML, Bristow RE, Diaz-Montes TP: Predictors of surgical outcome and survival among elderly women diagnosed with ovarian and primary peritoneal cancer. Gynecol Oncol 2006, 103:1048-1053.

36. Parker D, Bradley C, Bogle SM, Lay J, Masood M, Hancock AK, Naylor B, Price JJ: Serum albumin and CA125 are powerful predictors of survival in epithelial ovarian cancer. Br J Obstet Gynaecol 1994, 101:888-893.
37. Small W, Carrara R, Danford L, Logemann JA, Cella D: Quality of life and nutrition in the patient with cancer. Integrating Nutrition into your Cancer Program 2002.

38. Marin Caro MM, Laviano A, Pichard C: Impact of nutrition on quality of life during cancer. Curr Opin Clin Nutr Metab Care 2007, 10:480-487.

39. Rogers SN, Lowe D, Brown JS, Vaughan ED: The relationship between length of stay and health-related quality of life in patients treated by primary surgery for oral and oropharyngeal cancer. Int J Oral Maxillofac Surg 2001, 30:209-215.

Pre-publication history

The pre-publication history for this paper can be accessed here: http:/wwwbiomedcentral.com/1471-2407/10/232/prepub

doi: $10.1186 / 1471-2407-10-232$

Cite this article as: Laky et al., Pretreatment malnutrition and quality of life association with prolonged length of hospital stay among patients with gynecological cancer: a cohort study BMC Cancer 2010, 10:232

\section{Submit your next manuscript to BioMed Central and take full advantage of:}

- Convenient online submission

- Thorough peer review

- No space constraints or color figure charges

- Immediate publication on acceptance

- Inclusion in PubMed, CAS, Scopus and Google Scholar

- Research which is freely available for redistribution
C) Biomed Central 